\title{
Frontières
}

\section{Les réactions de deuil des parents lors d'une perte périnatale}

\section{Chantal Verdon}

Volume 16, numéro 2, printemps 2004

Deuil, blessure vive

URI : https://id.erudit.org/iderudit/1074114ar

DOI : https://doi.org/10.7202/1074114ar

Aller au sommaire du numéro

\section{Éditeur(s)}

Université du Québec à Montréal

\section{ISSN}

1180-3479 (imprimé)

1916-0976 (numérique)

Découvrir la revue

\section{Citer cet article}

Verdon, C. (2004). Les réactions de deuil des parents lors d'une perte périnatale. Frontières, 16(2), 38-42. https://doi.org/10.7202/1074114ar

\section{Résumé de l'article}

Cet article présente des résultats de recherche portant sur l'identification d'indicateurs de santé qui peuvent influencer l'intensité des réactions de deuil chez des parents qui vivent la mort de leur bébé en période périnatale. Ce type de perte provoque des remises en question qui semblent influencer la santé des parents endeuillés. Cette étude descriptive avait pour but de vérifier le rôle du sens donné à la vie sur la perception de la perte et les réactions de deuil des parents, deux mois et six mois après la perte de leur bébé. Les résultats mettent en évidence l'importance de soutenir les familles lorsque celles-ci présentent un sens donné à la vie moins positif, une perception de la perte plus négative et un faible soutien social. Il est recommandé d'explorer davantage l'expérience du père face au deuil d'un bébé et de lui accorder autant d'importance qu'à celle de la mère dans les suivis offerts. Pour le milieu clinique, on suggère d'explorer davantage les indicateurs de santé pour adapter les suivis selon les besoins des familles. 


\section{Résumé}

Cet article présente des résultats de recherche portant sur l'identification d'indicateurs de santé qui peuvent influencer l'intensité des réactions de deuil chez des parents qui vivent la mort de leur bébé en période périnatale. $\mathrm{Ce}$ type de perte provoque des remises en question qui semblent influencer la santé des parents endeuillés. Cette étude descriptive avait pour but de vérifier le rôle du sens donné à la vie sur la perception de la perte et les réactions de deuil des parents, deux mois et six mois après la perte de leur bébé. Les résultats mettent en évidence l'importance de soutenir les familles lorsque celles-ci présentent un sens donné à la vie moins positif, une perception de la perte plus négative et un faible soutien social. Il est recommandé d'explorer davantage l'expérience du père face au deuil d'un bébé et de lui accorder autant d'importance qu'à celle de la mère dans les suivis offerts. Pour le milieu clinique, on suggère d'explorer davantage les indicateurs de santé pour adapter les suivis selon les besoins des familles.

Mots clés: deuil périnatal - indicateurs de santé - sens de la vie - perception soutien social.

\begin{abstract}
This article presents research results on the identification of health indicators which can influence the intensity of mourning reactions in parents who experience the death of their baby during the perinatal period. This type of loss provokes major existential questions, which seem to impact the health of grieving parents. The purpose of this descriptive study was to verify the role that the meaning of life has on the perception of loss and the reactions of the parents two and six months after the loss of their baby. The results highlight the importance of supporting families who demonstrate a less positive meaning of life, a stronger negative perception of the loss and a weak social support network. Greater exploration of the father's reactions to the death of the baby is recommended as well as the necessity to grant him equal importance with the mother in the offered followups. For the clinical milieu, we suggest the exploration of health indicators to better adapt the follow-ups to the family's needs.
\end{abstract}

Key words: perinatal death - health indicators - meaning of life perception-social support.

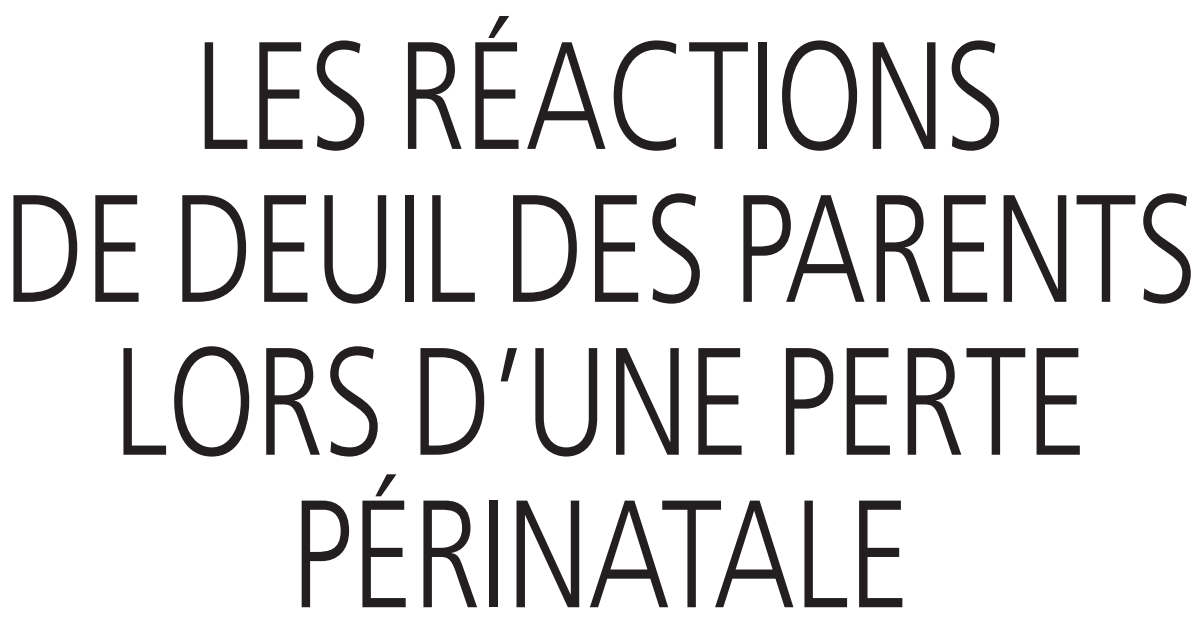

Chantal Verdon, B.Sc.Inf. chargée d'enseignement, Faculté de sciences infirmières, Université Laval.

L'expérience que vivent les parents confrontés à la mort de leur bébé lors d'une perte périnatale (soit pendant la grossesse, lors de l'accouchement ou de quelques jours à quelques semaines suivant la naissance du nourrisson) est pénible. Le but de cette recherche est de mieux saisir les éléments qui peuvent influencer la santé des familles endeuillées. Les résultats ont permis de proposer des indicateurs de santé susceptibles de guider les intervenants dans leurs interventions. Le sens donné à la vie, la perception de la perte et le soutien social font partie des concepts abordés dans cette étude.

\section{REMISE EN QUESTION DU SENS DE LA VIE LORS D'UNE PERTE PÉRINATALE}

Le sens de la vie est ce questionnement qui peut amener la personne à voir différemment sa souffrance et ainsi générer des changements positifs ou négatifs dans sa vie (Wheeler, 2001 ; Davis et al., 2000 ; Frankl, 1988). Lors d'une perte périnatale, les familles doivent s'adapter au vide laissé par le départ du bébé. Le processus de deuil qui s'enclenche se manifeste par des réactions de deuil d'intensité variable. On observe que plusieurs de ces familles cherchent un sens à la vie et que cette quête de sens semble influencer l'intensité des réactions de deuil, indépendamment du laps de temps écoulé depuis la perte du bébé. Dans cet ordre d'idées, Davis et ses collaborateurs (2000) exposent certaines hypothèses quant au concept de sens; la personne peut chercher un sens à l'événement, réfléchir sur la compréhension d'un tel événement dans sa vie ou encore sur ce que cette perte peut engendrer comme changements dans sa vie. Ce dernier élément fait partie de la perspective existentielle qui explique le questionnement centré sur la vie à la suite de la perte, et qui alors peut engendrer des changements, des réinvestissements, des nouveaux buts et objectifs chez la personne.

Chez la plupart des personnes endeuillées, à des moments différents dans leur processus de deuil, on observe que ce questionnement semble se produire même s'il n'aboutit pas toujours à des réponses positives (Neuman, 1995). Cette recherche de sens à la vie revêt un caractère philosophique et spirituel. Neuman propose un cadre de référence pour mieux l'expliquer; nous nous en sommes inspirée pour cette étude. Selon cette auteure, avoir la capacité de trouver un sens à la vie représente une force personnelle qui joue un rôle important dans la résolution du deuil. Pour d'autres auteurs, la recherche de sens à la vie semble être essentielle dans le processus de deuil, puisqu'elle permet aussi de trouver un sens à la souffrance et au chagrin causés par la perte (Chalifour, 2000 ; Davis et al., 2000 ; Frankl, 1988).

Cette force intérieure, en continuel développement, est influencée par les expériences de vie et devrait permettre à l'intervenant de jouer un rôle de prévention dans l'observation des signes précurseurs d'un deuil difficile. Par exemple, en explorant les ressources personnelles, telles que la recherche de sens à la vie à la suite d'une perte périnatale, l'intervenant pourrait mieux cibler les familles plus à risque, nécessitant une aide et un soutien professionnel. 


\section{LA PERCEPTION DE LA PERTE}

La perception se définit comme étant la représentation consciente que se fait la personne d'une situation à partir des sensations qu'elle vit (Maubourguet, 1995). La perception des parents et leurs réactions de deuil sont liées entre elles pour ainsi fournir des explications importantes sur leur processus d'adaptation. Ce processus dynamique est en changement constant dans le temps, puisqu'il est influencé par la situation de stress, les ressources dont dispose la personne et les différentes stratégies disponibles (Lazarus et Folkman, 1984). Aussi, le fait de banaliser la perte précoce pendant la grossesse rend les parents plus confus dans leur propre perception de l'événement qu'ils vivent (Hutti, 1992). Cet exemple peut expliquer l'existence d'un lien entre la perception de la perte, les réactions de deuil et le soutien social reçu. On croit que ce concept est un facteur constant d'influence sur les réactions des mères et des pères.

\section{LES RÉACTIONS DE DEUIL DES MÈRES ET DES PÈRES}

Vivre la perte d'un bébé pendant la période périnatale a des répercussions importantes pour chaque famille. Les réactions de deuil sont nombreuses et touchent plusieurs dimensions dans la vie des parents. Les différences entre les réactions de deuil des mères et des pères révèlent un aspect important du deuil périnatal. Les couples qui ne vivent pas les réactions de deuil au même moment et avec la même intensité (Vance et al., 1995; Lang et Gottlieb, 1993) révèlent des différences fondamentales qui créent un déséquilibre important dans la relation entre les conjoints. De plus, le temps et la durée des réactions de deuil (Lin et Lasker, 1996) inquiètent la plupart des parents. Ceux-ci entendent un discours qui prône l'importance de s'adapter rapidement aux conditions de leur nouvelle vie sans bébé. Cette constatation explique l'intérêt profond avec lequel nous avons exploré, dans cette étude, les réactions de deuil et leurs différences entre les conjoints et dans le temps, à la suite d'une perte périnatale.

\section{LE SOUTIEN SOCIAL}

Le soutien social semble être un élément très important pour expliquer l'intensité des réactions de deuil. Dans le discours des parents, il semble que plus le temps s'écoule depuis la perte, plus les parents se sentent isolés. Ils disent se sentir seuls et ne pas savoir à qui parler de leur bébé décédé et de leur souffrance. Les gens de l'entourage poursuivent leur vie et n'y font plus allusion. De plus, au moment de la perte et dans les jours qui suivent, les paroles, les gestes et les attitudes de la famille ou des profes- sionnels sont souvent retenus intégralement par les parents et constituent en soi des souvenirs qui les aideront ou non à s'adapter à la perte (Robinson, Baker et Nackerud, 1999).

Les discours recueillis confirment que ce vécu est important pour les familles endeuillées, que cette dimension revêt une grande signification pour expliquer les réactions de deuil des parents. Un deuil non reconnu suffisamment par la famille élargie et les amis semble être plus difficile à gérer.

\section{PRÉSENTATION DE LA RECHERCHE}

Le but de cette étude descriptive est de vérifier le rôle du sens de la vie sur la perception des parents et leurs réactions de deuil deux mois et six mois après la perte de leur bébé. Certains facteurs d'influence, identifiés préalablement dans la littérature, ont été mis en relation tels que le soutien social reçu par l'entourage et le soutien conjugal. D'autres caractéristiques reliées à l'histoire de la perte ont également été mises en relation avec ces concepts tels que le type de perte, le caractère subit ou anticipé de la perte, la présence des autres enfants au moment de la perte et une grossesse subséquente.

Cette étude veut vérifier l'hypothèse suivante : lorsque les parents donnent un sens positif à la vie, la perception de la perte du bébé est moins négative et leurs réactions de deuil sont moins intenses; cette hypothèse est en lien avec les différents facteurs d'influence. De plus, l'existence des différences entre les mères et les pères pour chacune des variables à l'étude, incluant les facteurs d'influence aux deux périodes dans le temps, soit deux mois et six mois après la perte du bébé, a été vérifiée.

Cette étude descriptive a été effectuée auprès de 56 couples, deux mois après la perte. Lors de la seconde entrevue, soit six mois après la perte, quatre d'entre eux se sont désistés. Les couples recrutés devaient être âgés de plus de 18 ans, comprendre et lire le français ou l'anglais, avoir perdu leur bébé au cours de la grossesse ou durant les 28 jours suivant sa naissance, habiter en couple et à moins de 100 kilomètres du lieu de l'étude. Ils devaient s'agir d'une première expérience de perte pour les deux parents. Les parents qui ont vécu une perte par interruption volontaire de grossesse ont été exclus de l'échantillon à cause du caractère différent de la perte.

Les couples ont d'abord répondu à un questionnaire sociodémographique; les données personnelles et autres informations recueillies touchent plus particulièrement la description de la perte. Ce questionnaire leur a été soumis deux mois après la perte. Le questionnaire administré lors de la seconde entrevue, quatre mois plus tard, permettait de savoir s'il y avait eu des changements significatifs dans leur vie, comme d'autres grossesses et/ou d'autres pertes périnatales.

Les parents qui ont participé à l'étude étaient âgés entre 19 et 47 ans ; l'âge moyen des mères était de 30 ans $(s=4,5)$ et celui des pères de 33 ans $(s=5,7)$. La plupart des couples sont Canadiens français. La moitié des parents ont un niveau de scolarité universitaire tandis que l'autre moitié est soit de niveau collégial (30) ou secondaire (21). Quant au revenu annuel familial, la médiane se situe dans la catégorie 60000 \$ à 79999 \$. Plus du tiers des couples (22) ont indiqué un revenu familial supérieur à 80000 \$. Lors de la deuxième entrevue, quatre couples avaient eu une augmentation dans leur revenu annuel familial. Plus de la moitié des parents, soit 34 couples, ont vécu une perte précoce $(>13$ semaines de gestation) dont plus de la moitié à 12 semaines de gestation. Quatorze couples ont perdu leur bébé de façon tardive ( $<13$ semaines de gestation à la naissance) et 8 autres couples pendant la période néonatale (entre un jour et 20 jours de vie). La moyenne de semaines de gestation est de 16,6 semaines $(s=10,0)$. De ces 56 couples, 27 d'entre eux avaient au moins un enfant au moment de la perte. Six mois plus tard, 14 couples avaient une autre grossesse en cours et deux couples avaient vécu une seconde perte entre les deux temps de mesure. Quatre mères et deux pères recevaient une aide professionnelle et participaient à des rencontres de groupe d'entraide lors de la première entrevue. À la deuxième rencontre, six mères et trois pères avaient consulté pour une aide professionnelle et trois mères seulement assistaient à des rencontres de groupe d'entraide.

Les couples ont été recrutés dans sept hôpitaux qui offrent des soins en périnatalité. Dans chaque centre participant, une infirmière attitrée téléphonait aux parents afin de sonder leur intérêt à recevoir de l'information concernant l'étude. Dans l'affirmative, leurs coordonnées étaient acheminées à la chercheuse qui leur adressait une lettre expliquant le but de l'étude, l'implication attendue ainsi que les détails de l'étude. Une relance téléphonique a été effectuée par la chercheuse pour répondre aux questions, confirmer la participation des parents et fixer une rencontre au domicile des parents, deux mois après la perte du bébé; les deux parents devaient être présents. Lors de cette rencontre, chaque parent a répondu individuellement au questionnaire; l'accent a été mis sur l'importance de répondre spontanément à chaque question. Une seconde lettre a été envoyée aux parents environ cinq mois et demi après la perte pour leur demander de 
confirmer leur participation. Une relance téléphonique a alors été effectuée pour fixer un second rendez-vous. Les parents ont été visités à nouveau, quatre mois plus tard,

\section{LES INSTRUMENTS DE MESURE}

\section{LE SENS DE LA VIE}

Le sens de la vie est la tendance de l'être humain à recadrer et mettre en perspective les répercussions d'une situation difficile, en changeant au niveau cognitif et émotionnel la façon de voir la situation, et conséquemment de trouver un but et un nouveau sens à l'existence (Lang et al., 2001). Le sens de la vie a été mesuré à l'aide des 16 items de la sous-échelle «sens de la vie » de l'instrument de mesure «Lang/Goulet Hardiness Scale» ou LGHS (Lang et Goulet, 1998). au sens de la vie pour permettre de mieux saisir cette variable dans le contexte d'une dent en encerclant leur degré d'accord ou de désaccord sur une échelle de Likert proposant 5 choix. Un score élevé indique un haut degré de sens de la vie.

\section{LA PERCEPTION}

La perception de l'événement correspond de façon théorique à l'évaluation cognitive primaire et secondaire de la borée par Lazarus et Folkman (1984). La Grille d'évaluation subjective des stresseurs (GESS) développée par Lemyre (1986) et réadaptée par Biron (1992) et Fillion (1993) a été utilisée. Cette grille sert à évaluer de façon subjective l'agent stresseur selon dix dimensions cognitives: les conséquences pour la deuxième mesure. Le tableau 1 expose les questions relatives étude quantitative. Les personnes réponthéorie du stress et de l'adaptation élapositives et négatives, la perte, le danger,

l'échec, les défis, le contrôle, la capacité de supporter la situation et l'importance qui lui est accordée (Fillion et al., 1996). Un score élevé indique une perception plus positive de l'événement.

\section{LES RÉACTIONS DE DEUIL}

Les réactions de deuil ont été définies comme étant : la colère, la culpabilité, la dépression et les préoccupations reliées à la perte. Cette variable est mesurée par l'Échelle de deuil périnatal «Perinatal Grief Scale » ou PGS (Toedter et al., 1988), où la version plus courte a été développée par Potvin, Lasker et Toedter (1989). Cette échelle comprend 33 éléments divisés en trois sous-échelles, soit le deuil actif, la difficulté de supporter le deuil et le désespoir. La version courte de cet instrument est équivalente à la version originale (Potvin et al., 1989).

\section{LE SOUTIEN CONJUGAL ET SOCIAL}

Les soutiens conjugal et social sont des facteurs d'influence retenus pour l'étude. Linstrument de mesure «Support Behaviors Inventory » (SBI), développé par Brown (1986) et basé sur House (1981), définit l'aspect social à travers les dimensions émotionnelles, évaluatives, informatives, instrumentales, et la perception subjective de la personne sur la qualité du soutien reçu. La particularité de cet instrument réside au niveau des sous-échelles qui mesurent à l'aide de 11 éléments chacune, les soutiens conjugal et social, c'est-à-dire celui offert par les autres membres de la famille. L'instrument a été traduit en français selon la méthode inversée et adaptée pour la population québécoise (Goulet, Polomeno et Harel, 1995).

Tableau 1 - QUESTIONS POSÉES SUR LE SENS DE LA VIE

\begin{tabular}{ll}
$\begin{array}{l}\text { Lorsque je vis des situations difficiles, } \\
\text { je peux à peine penser à autre chose. }\end{array}$ & $\begin{array}{l}\text { Je m'imagine difficilement que la vie puisse } \\
\text { être excitante. }\end{array}$ \\
\hline $\begin{array}{l}\text { Je réalise que je suis une personne plus forte } \\
\text { que je pensais. }\end{array}$ & $\begin{array}{l}\text { Je crois que vivre des situations difficiles } \\
\text { renforce mes croyances religieuses. }\end{array}$ \\
\hline $\begin{array}{l}\text { Vivre des situations difficiles m'a aidé(e) } \\
\text { à reconnaître mes vrai(e)s ami(e)s. }\end{array}$ & $\begin{array}{l}\text { J'ai souvent l'impression de ne pas } \\
\text { me connaître. }\end{array}$ \\
\hline $\begin{array}{l}\text { Je crois que je peux regagner un sentiment } \\
\text { de paix intérieure. }\end{array}$ & $\begin{array}{l}\text { La plupart du temps, la vie ne vaut pas } \\
\text { la peine d'être vécue. }\end{array}$ \\
\hline $\begin{array}{l}\text { Je crois que je gâche une grande partie } \\
\text { de ma vie en faisant des choses } \\
\text { qui m'importent peu. }\end{array}$ & $\begin{array}{l}\text { Je crois qu'un des meilleurs moyens } \\
\text { de prendre une situation difficile } \\
\text { est d'essayer de la voir de différentes façons. }\end{array}$ \\
\hline J'apprécie vraiment ce que j'ai. & $\begin{array}{l}\text { Je crois que je fais une différence dans la vie } \\
\text { d'autres personnes. }\end{array}$ \\
\hline Je réalise comment la vie est précieuse. & $\begin{array}{l}\text { J'ai tendance à laisser des situations } \\
\text { particulières envahir tous les autres aspects } \\
\text { de ma vie. }\end{array}$ \\
\hline $\begin{array}{l}\text { Je crois que vivre des situations difficiles } \\
\text { rend ma spiritualité plus intense. }\end{array}$ & $\begin{array}{l}\text { Parfois, je crois que Dieu m'a abandonné. } \\
\end{array}$
\end{tabular}

\section{LES RÉSULTATS}

La validation des hypothèses s'est faite à l'aide d'analyses de corrélations de Pearson, de régressions et d'analyses de variance à mesures répétées sur deux ou trois facteurs selon le cas. Le seuil de signification statistique pour tous les tests a été déterminé à alpha $<0,05$.

\section{LA RELATION ENTRE LE SENS DE LA VIE, LA PERCEPTION ET LES RÉACTIONS DE DEUIL DES PARENTS}

Les résultats permettent de constater que le sens donné à la vie et la perception de la perte agissent comme indicateurs de santé et influencent les réactions de deuil des parents qui perdent un bébé en période périnatale.

Plus précisément chez les mères, leurs réactions de deuil sont moins intenses lorsqu'elles réussissent à donner un sens positif à la vie malgré la perte du bébé. De plus, leur perception de la perte influence l'intensité des réactions de deuil. Ces résultats confirment le caractère positif de la quête de sens à la vie et la façon de voir l'expérience et le processus de deuil. Selon le cadre conceptuel proposé par Neuman (1995), le parent qui trouve un nouveau sens à sa vie, en puisant dans ses ressources personnelles, voit la situation différemment, et rétablit alors son équilibre et vit des réactions de deuil moins intenses. Ces résultats viennent appuyer de façon empirique les écrits théoriques de plusieurs auteurs (Chalifour, 2000 ; Frankl, 1988 ; Bowlby, 1982). La plupart des études réalisées sur ce sujet sont de nature qualitative (Dyregrov et Dyregrov, 1999; Braun et Berg, 1994). Seule l'étude de Davis et ses collaborateurs (2000) utilise un devis semblable à celui de la présente étude et leurs résultats sont aussi comparables aux nôtres. Après avoir analysé les données recueillies auprès de 124 parents dont $79 \%$ sont des mères, Davis et ses collaborateurs soulignent le rôle du sens de la vie sur les symptômes de deuil en réduisant la détresse émotionnelle. Ces auteurs postulent que la recherche de sens à la vie permet de mieux s'ajuster à la perte et de se reconstruire une perspective existentielle. Neuman (1995) poursuit sur cette lancée, en abordant la reconstitution : la personne doit puiser en elle les ressources nécessaires à reconstruire un sens pour s'adapter à la perte.

On retrouve les mêmes constats chez les pères, lorsque six mois se sont écoulés depuis la perte du bébé. Les ressources personnelles qui prédisent l'intensité des réactions après la perte du bébé chez les pères peuvent prendre d'autres formes que la recherche de sens à la vie. Par exemple, les pères sont plus influencés par le soutien 
conjugal qui, lorsque satisfaisant, tend à expliquer une perception plus positive de la perte. Les données portant sur le soutien conjugal n'ont rien révélé chez les mères.

\section{LE SOUTIEN SOCIAL ET SON IMPACT SUR LES RÉACTIONS DE DEUIL ET LE SENS DONNÉ À LA VIE CHEZ LES PARENTS}

Le soutien social reçu de la part des personnes significatives, autres que le conjoint, influence le sens donné à la vie et l'intensité des réactions de deuil, plus particulièrement chez les mères. Plus elles reçoivent du soutien, plus elles donnent un sens positif à la vie et réagissent moins intensément. Chez les pères, on note qu'ils se sentent moins soutenus socialement que les mères, tant deux mois que six mois après la perte du bébé. Ceci appuie d'autres écrits (Pudiffoot et Johnson, 1997 ; Zeanah et al., 1995) qui mentionnent le peu de reconnaissance et de soutien social offert aux pères. Cependant, considérant l'importance du rôle que joue le père pour prendre soin de sa conjointe, on peut penser que celui-ci met son deuil en attente et réagit alors différemment de la mère. De plus, ces différences peuvent relever du processus de socialisation puisqu'on valorise davantage une répression des émotions chez les hommes.

\section{LES DIFFÉRENCES ENTRE LES MÈRES ET LES PÈRES}

Il existe des différences entre les mères et les pères en ce qui a trait à l'intensité de leurs réactions de deuil, leur perception de la perte et la satisfaction manifestée à l'égard du soutien social reçu. Les mères présentent des réactions de deuil plus intenses que les pères, et ce, aux deux temps de mesure. Cela peut s'expliquer partiellement par leur perception plus négative de l'événement. Nos résultats vont dans le même sens que ceux des études antérieures mesurant les différences entre les mères et les pères au niveau des réactions de deuil (Dyregrov et Dyregrov, 1999; Vance et al., 1995 ; Lang et Gottlieb, 1993) ou quant à la perception de la grossesse entre les conjoints (Vance et al., 1995) et la perception de la perte du bébé (Hutti, 1992). Une autre explication plausible concerne l'instrument de mesure davantage utilisé auprès d'échantillons féminins; peu d'études ont été réalisées uniquement auprès d'hommes endeuillés.

\section{LES DIFFÉRENCES DANS LE TEMPS APRÈS LA PERTE DU BÉBÉ}

Quant aux différences face aux variables à l'étude dans le temps, on note que les parents obtiennent des résultats différents

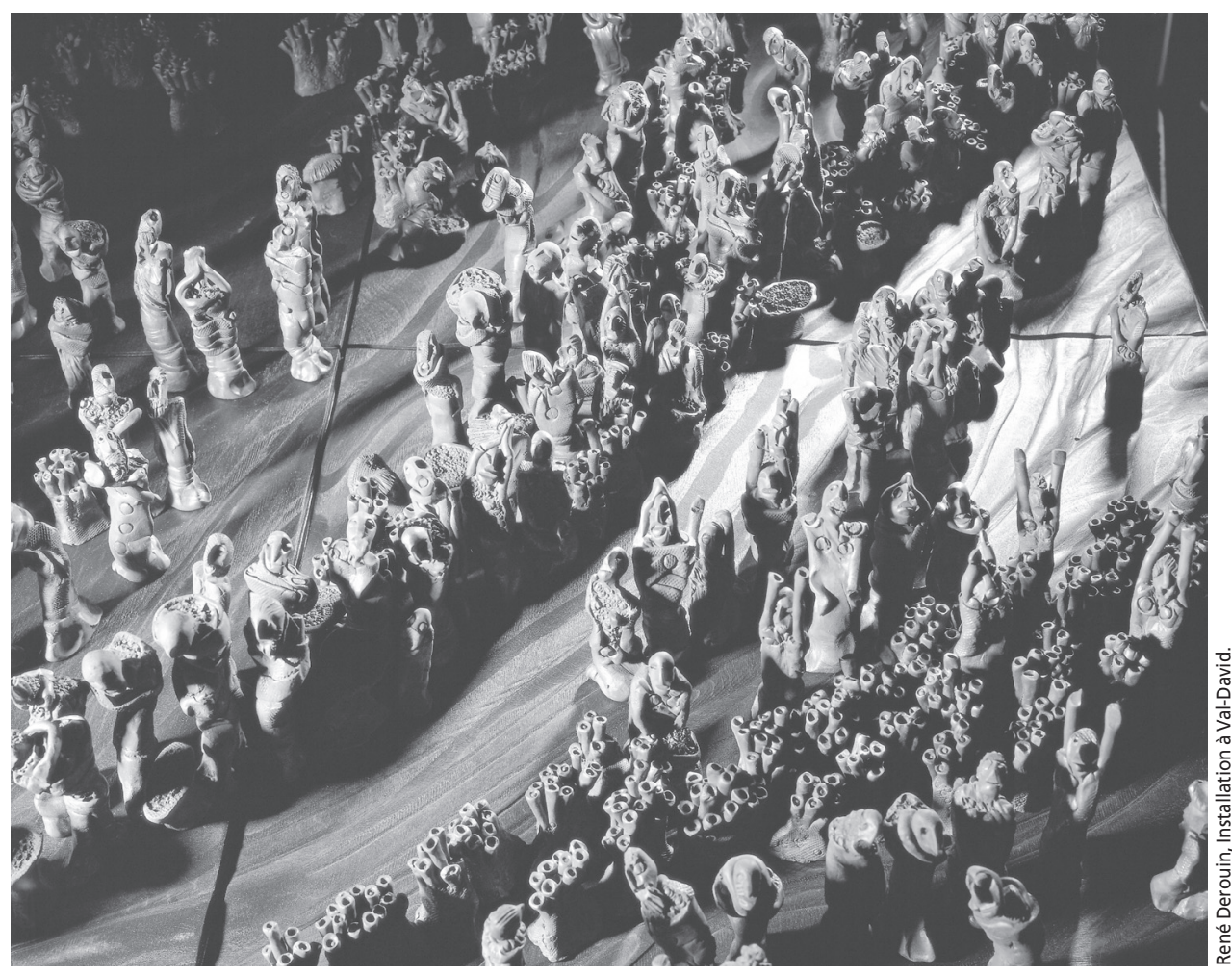

seulement pour l'intensité des réactions de deuil. Les réactions de deuil sont moins intenses six mois après la perte du bébé qu'elles ne l'étaient deux mois après la perte.

\section{LES AUTRES FACTEURS D'INFLUENCE}

Pour les autres facteurs d'influence, nos résultats ont démontré que le type de perte influence les réactions de deuil des parents. Ce constat tend à démontrer que plus la perte est précoce moins les réactions de deuil sont intenses chez les parents, confirmant ainsi d'autres résultats publiés auparavant. Toutefois, le type de perte n'influence pas la perception de l'événement, ce qui peut vouloir dire que de perdre un bébé tôt au cours de la grossesse ne permet pas de percevoir plus positivement la situation. De plus, perdre un bébé pendant la grossesse ou après la naissance n'influence pas la façon de donner un sens à la vie. Ces résultats permettent de questionner la relation existant entre le type de perte et les réactions de deuil.

Avoir d'autres enfants au moment de la perte influence les variables suivantes: le sens donné à la vie, la perception de la perte et les réactions de deuil des parents. Les parents qui perdent leur premier bébé obtiennent des scores de sens à la vie significativement inférieurs à ceux qui ont déjà un enfant, perçoivent l'événement plus négativement et ont des réactions de deuil plus intenses. Ces résultats corroborent ceux de Wheeler (1994) qui soutient que perdre son unique enfant est beaucoup plus difficile comparativement à ce qui se passe lorsque les parents ont déjà d'autres enfants. Ces résultats corroborent aussi les écrits théoriques (Fréchette, 1997 ; Garel et Legrand, 1995) qui stipulent, entre autres, que les mères vivent des craintes reliées à la possibilité de ne pas pouvoir enfanter. Les parents font alors le deuil d'être parents, ce qui peut expliquer un sens de la vie moins positif, une perception plus négative et une intensité des réactions de deuil plus grande. Néanmoins, les parents qui ont des enfants au moment de la perte nous confirment que leurs obligations parentales ne leur permettent pas de se laisser aller intensément dans leurs réactions de deuil ; ce qui peut nous guider quant aux résultats obtenus.

Le caractère subit ou anticipé de la perte ainsi qu'une grossesse subséquente n'ont pas influencé les résultats face au sens donné à la vie, à la perception de la perte et à l'intensité des réactions de deuil. Nous croyons que certains facteurs, non mentionnés dans cette étude, ont pu influencer la réponse des parents tels que des variables étrangères comme les rituels effectués à la suite de la perte, les croyances et les valeurs de chaque personne, les expériences antérieures de perte, le bagage personnel et la désirabilité sociale.

\section{RETOMBÉES POUR LA \\ RECHERCHE ET LA CLINIQUE}

L'interprétation des résultats propose des indicateurs de santé pour mieux comprendre la réalité des parents qui font face à un deuil périnatal. Les ressources personnelles, comme la recherche de sens à la vie, ou les ressources puisées dans le milieu de 
vie, comme le soutien social, permettent de mieux gérer le deuil et aident les parents à s'adapter à la perte de leur bébé.

Une attention particulière devrait être apportée aux réactions des pères ainsi qu'à la perception qu'ils ont de la perte. Leur satisfaction face au soutien social reçu devrait être explorée au cours de la période suivant la perte, au moins six mois après l'événement. Les intervenants pourraient prévoir des rencontres individuelles avec chaque famille ; la mère et le père seraient invités à y participer dès le décès du bébé.

En recherche, on devrait explorer davantage les besoins et l'expérience du deuil chez les pères. Des études comparatives entre les pères qui reçoivent un soutien et ceux qui n'en ont pas pourraient contribuer à comprendre l'impact d'un tel événement chez les hommes et le rôle que joue le soutien social. Élaborer des instruments de mesure spécifiques aux réactions des pères permettrait une mesure plus précise de ce qu'ils vivent puisque la plupart des instruments ont surtout été élaborés auprès d'échantillons comparés de femmes.

Il demeure important que les interventions ayant pour but d'explorer les ressources personnelles des parents et celles auxquelles ils ont accès dans leur milieu de vie permettent d'identifier les familles plus à risque de vivre un deuil qui pourrait compromettre leur santé. Les résultats de cette étude peuvent contribuer à modifier les interventions auprès des familles qui perdent un bébé en mettant en évidence les éléments à explorer pour les aider à vivre leur deuil. Les réflexions des parents sur leur quête de sens à la vie peuvent les encourager à nommer les nombreux changements occasionnés par la perte du bébé et leur permettre d'identifier les éléments pertinents qui touchent le sens de la vie comme les croyances, les valeurs et les priorités bouleversées par la mort du bébé. On pourrait alors mieux cibler leurs besoins et peut-être prévenir les problèmes en identifiant le niveau de risque associé à des réactions de deuil intenses. Ainsi, il devient impératif d'élaborer des suivis personnalisés de soutien et d'accompagnement pour chaque parent qui présente un ou des indicateurs de santé tels un faible soutien social, une perception négative de sa perte, un sens donné à la vie difficilement positif sans changements au quotidien, tout en portant une attention marquée aux parents qui perdent leur seul enfant. Ces observations devraient amener les intervenants à prendre conscience de l'importance d'effectuer une démarche de relance auprès de ces parents et de leur offrir un accompagnement plus adapté à leur situation de deuil.

\section{Bibliographie}

BIRON, C. (1992). La détresse émotionnelle chez les couples infertiles: aspects contextuels et subjectifs des stresseurs, Thèse de doctorat, Université Laval, Québec.

BOWLBY, J. (1982). «Attachment and loss: Retrospect and prospect», The American Journal of Orthopsychiatry, vol. 52, p. 664-678.

BRAUN, M.J. et D.H. BERG (1994). « Meaning reconstruction in the experience of parental bereavement», Death Studies, vol. 18, p. $105-129$.

BROWN, M.A. (1986). « Social support during pregnancy: A unidimensional or multidimensional construct? », Nursing Research, vol. 35, $\mathrm{n}^{\circ} 1$, p. 4-9.

CHALIFOUR, J. (2000). « L'accompagnement des personnes endeuillées ", dans Lintervention thérapeutique, stratégies d'intervention 2, Montréal, Gaëtan Morin Éditeur, p. 208-252.

DAVIS, C.G., C.B. WORTMAN, D.R. LEHMAN et R.C. SILVER (2000). «Searching for meaning in loss: Are clinical assumptions correct?», Death Studies, vol. 24, p. 447-540.

DYREGROV, A. et K. DYREGROV (1999). "Long-term impact of sudden infant death: a 12-to 15-year follow-up », Death Studies, vol. 23, p. 635-661.

FILLION, L. (1993). Évaluation cognitive, état de stress et immunité lors d'un diagnostic de tumeur, thèse de doctorat, Université Laval, Québec.

FILLION, L., B.E. HURWITZ, M.A. FLETCHER, J.S. GRAVES, N.G. KLIMAS et N. SCHNEIDERMAN (1996, March). Reliability and validity of the SARS in assessing the cognitive appraisal of HIV status, Paper presented at the The Fourth International Congress of Behavioral Medicine, Washington, D.C.

FRANKL, V.E. (1988). Découvrir un sens à sa vie: avec la logothérapie, Montréal, Éditions de l'Homme.

FRÉCHETTE, L. (1997). «Deuil à la suite d'accidents de procréation et de décès de nourrissons », Frontières, vol. 9, nº 3, p. 10-14.

GAREL, M. et H. LEGRAND (1995). Une fausse-couche et après, Paris, Albin Michel.

GOULET, C., V. POLOMENO et F. HAREL (1995). Translation and Psychometric Evaluation of Research Instrument, Rapport final de recherche, Faculté des sciences infirmières, Université de Montréal.

HOUSE, J. (1981). Work Stress and Social Support, Menlo Park, California, AddisonWesley.

HUTTI, M.H. (1992). « Parents' perception of the miscarriage experience», Death Studies, vol. 16, p. 401-415.

LANG, A. et L. GOTTLIEB (1993). «Parental grief reactions and marital intimacy following infant death », Death Studies, vol. 17, n 3, p. 233-255.

LANG, A. et C. GOULET (1998). Traduction de l'instrument «Perinatal grief scale», Document non publié, Université de Montréal.
LANG, A., C. GOULET, M. AITA, V. GIGUERE, H. LAMARRE et E. PERREAULT (2001). "Weathering the storm of perinatal bereavement via hardiness ", Death Studies, vol. 25, p. 497-512.

LAZARUS, R. et S. FOLKMAN (1984). Stress, Appraisal and Coping, New York, Springer.

LEMYRE, L. (1986). Stress et appréhension cognitive, Thèse de doctorat, Université Laval, Québec.

LIN, S.X. et J.N. LASKER (1996). " Patterns of grief reaction after pregancy loss », American Journal of Orthopsychiatry, vol. 66, $\mathrm{n}^{\circ} 2$, p. 262-271.

MAUBOURGUET, P. (1995). Le Petit Larousse illustré, Dictionnaire encyclopédique, Larousse, Paris.

NEUMAN, B. (1995). The Neuman Systems Model (3 ${ }^{\text {e }}$ éd.), Stanford, Connecticut, Appleton \& Lange.

POTVIN, L., J. LASKER et L. TOEDTER (1989). «Measuring grief: A short version of the Perinatal Grief Scale », Journal of Psychopathology and Behavioral Assessment, vol. $11, \mathrm{n}^{\circ} 1$, p. 29-45.

PUDIFFOOT, J.E. et M.P. JOHNSON (1997). «The legitimacy of grieving: The partner's experience at miscarriage », Social Science Medicine, vol. 45, $\mathrm{n}^{\circ}$ 6, p. 837-845.

ROBINSON, M., L. BAKER et L. NACKERUD (1999). "The relationship of attachment theory and perinatal loss ", Death Studies, vol. 23, p. 257-270.

THEUT, S.K., F.A. PEDERSEN, M.J. ZASLOW, R.L. CAIN, B.A. RABINOVICH et J.M. MORIHISA (1989). «Perinatal loss and parental bereavement ", American Journal of Psychiatry, vol. 146, n ${ }^{\circ}$, p. 635-639.

TOEDTER, L.J., J.N. LASKER et J.M. ALHADEFF (1988). "The Perinatal Grief Scale: Development and initial validation », American Journal of Orthopsychiatry, vol. 58, $\mathrm{n}^{\circ}$ 3, p. 435-449.

VANCE, J.C., F.M. BOYLE, J.M. NAJMAN et M.J. THEARLE (1995). «Gender differences in parental psychological distress following perinatal death or sudden infant death syndrome ", British Journal of Psychiatry, vol. 167, p. 806-811.

VERDON, C. (2002). Sens de la vie, perception et réactions de deuil de parents suite à une perte périnatale, Mémoire de maîtrise, Faculté des sciences infirmières, Université de Montréal.

WHEELER, I. (2001). « Parental bereavement: the crisis of meaning», Death Studies, vol. 23, p. 51-66.

WHEELER, I. (1994). « The role of meaning: and purpose in life in bereaved parents associated with a self-help group : Compassionate friends ", Omega, Journal of Death \& Dying, vol. $28, n^{\circ} 4$, p. 261-271.

ZEANAH, C.H., B. DANIS, L. HISHBERG et L. DIETZ (1995). "Initial adaptation in mothers and fathers following perinatal loss ", Infant Mental Health Journal, vol. 16, $\mathrm{n}^{\circ} 2$, p. 80-93. 